\title{
Radiation Therapy-Induced Phrenic Neuropathy Mimicking Metastasis to the Diaphragmatic Crus on ${ }^{18}$ F-FDG PET/CT
}

\author{
Prashant Jolepalem ${ }^{1}$, Jane Y. Hwang ${ }^{2}$, and C.Y. Oliver Wong ${ }^{1}$ \\ ${ }^{1}$ Department of Diagnostic Radiology and Molecular Imaging, Oakland University William Beaumont School of Medicine and \\ Beaumont Health System, Royal Oak, Michigan; and ${ }^{2}$ School of Medicine, Oakland University William Beaumont School of Medicine \\ and Beaumont Health System, Royal Oak, Michigan
}

\begin{abstract}
Benign unilateral uptake in the diaphragmatic crus is a relatively uncommon finding on ${ }^{18} \mathrm{~F}-\mathrm{FDG}$ PET/CT that can mimic the appearance of malignancy in patients with known cancer, as illustrated in this patient with neuroendocrine carcinoma. It is important for the PET interpreter to recognize this finding and attribute it to benign uptake based on the patient's history and symptoms at the time of the scan. Correlation with the findings of other available imaging modalities is also important to characterize focal uptake in unusual locations.
\end{abstract}

Key Words: correlative imaging; oncology; PET/CT; ${ }^{18} \mathrm{~F}-\mathrm{FDG}$ PET; abnormal variant uptake; diaphragmatic crus

J Nucl Med Technol 2014; 42:68-69

DOI: 10.2967/jnmt.113.130096

I $n$ its ability to stage cancers, evaluate response to treatment, and predict patient prognosis, ${ }^{18} \mathrm{~F}-\mathrm{FDG}$ PET has proven value. However, interpretation can be made challenging by physiologic, inflammatory, or normal variant activity that can mimic the appearance of malignancy (1). This case demonstrates a relatively uncommon abnormal variant of benign unilateral uptake in the diaphragmatic crus (2). It is important for the PET interpreter to recognize this finding and attribute it to benign uptake based on the patient's history and symptoms at the time of the scan.

\section{CASE REPORT}

A 63-y-old woman diagnosed with neuroendocrine carcinoma of the right lung apex in 2003 presented for a restaging PET/CT examination 1 mo after chemoradiation therapy.

The ${ }^{18}$ F-FDG PET scan demonstrated focally increased uptake in the right diaphragmatic crus (maximum standardized uptake value, 4.3) that was not present on her PET scan

\footnotetext{
Received Jul. 29, 2013; revision accepted Sep. 23, 2013.

For correspondence or reprints contact: Prashant Jolepalem, William

Beaumont Hospital, 3601 W. 13 Mile Rd., Royal Oak, MI 48073.

E-mail: pjolepalem@gmail.com

Published online Jan. 27, 2014.

COPYRIGHT (c) 2014 by the Society of Nuclear Medicine and Molecular Imaging, Inc.
}

performed 1 y previously but was present on a restaging study performed 2 y previously (Fig. 1). Because the rest of the scan was essentially negative for uptake, this finding needed further investigation. Review of the chart revealed that the patient had not been undergoing radiation therapy at the time of the last study but had received treatment within 3 mo of her previous PET study, which had demonstrated the unilateral diaphragmatic crus uptake. Additionally, the scout view of a recently performed diagnostic CT scan demonstrated right hemidiaphragm elevation (Fig. 2), which is highly suggestive of paralysis secondary to phrenic neuropathy (3).

Given the temporal relationship between radiation therapy and the right diaphragmatic crus uptake on ${ }^{18} \mathrm{~F}-\mathrm{FDG}$ PET and hemidiaphragm elevation, one could surmise that this was benign activity. Because the activity was on the paralyzed side of the diaphragm, radiation-induced phrenic nerve neuropathy was the likely etiology.

Currently, the patient does not demonstrate any evidence of recurrent malignancy by imaging or tumor markers. She is being monitored by serial imaging at a reduced interval and has discontinued radiation therapy.

\section{DISCUSSION}

The diaphragmatic crus is an uncommon site of benign physiologic ${ }^{18}$ F-FDG PET uptake that can be caused by hyperventilation, hiccups, or restrictive pulmonary disease (1). However, in such cases, the uptake is typically bilateral. Unilateral diaphragmatic uptake is most often due to diaphragmatic paralysis $(2,4)$. When the uptake is contralateral to the paralyzed hemidiaphragm, the etiology is a compensatory increase in the work of the functioning side (4). When the uptake is on the ipsilateral side, phrenic nerve neuropathy should be suspected. In the setting of malignancy, secondary effects of external-beam radiation can cause hemidiaphragmatic paralysis via fibrotic changes or induction of phrenic neuropathy (5). Phrenic neuropathy is reversible, but recovery is slow (3).

Because the transient focal unilateral uptake described in this patient demonstrated a temporal relationship with radiation treatments and was ipsilateral to the paralyzed and elevated hemidiaphragm, radiation-induced left phrenic neuropathy could be reasonably suspected. 


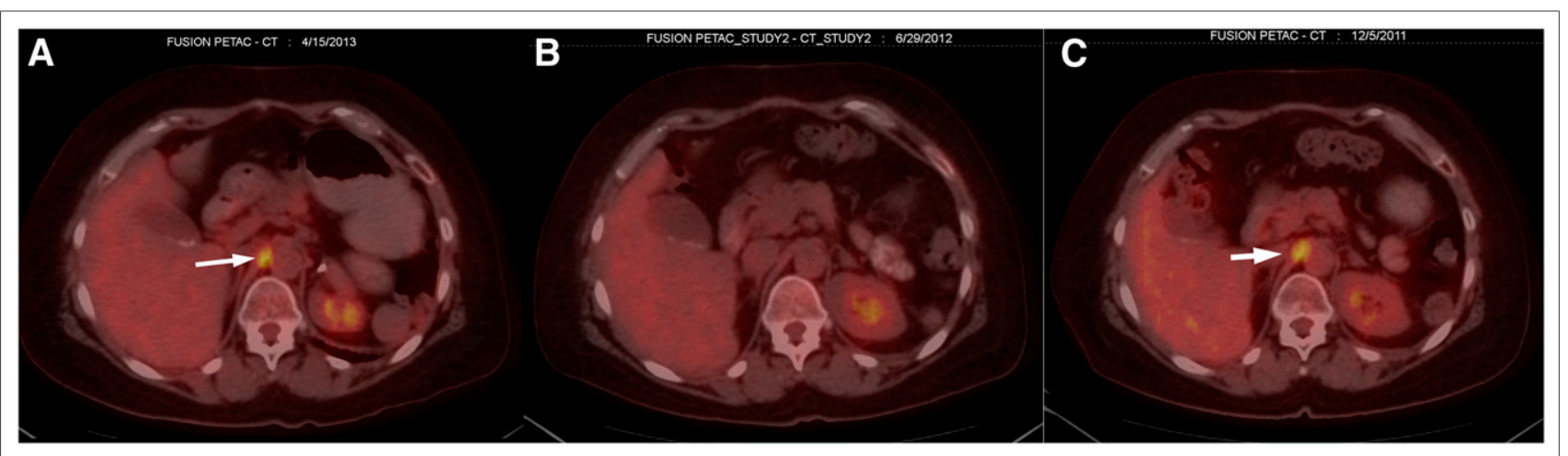

FIGURE 1. Three PET/CT fusion images taken approximately 1 y apart. Current study (A) and study acquired 2 y previously (C) were both performed within 3 mo of external-beam radiation therapy, and both demonstrate increased uptake (arrows) at right diaphragmatic crus. Patient had not received any recent radiation therapy at time study acquired 1 y previously (B), which did not show any uptake at right crus.

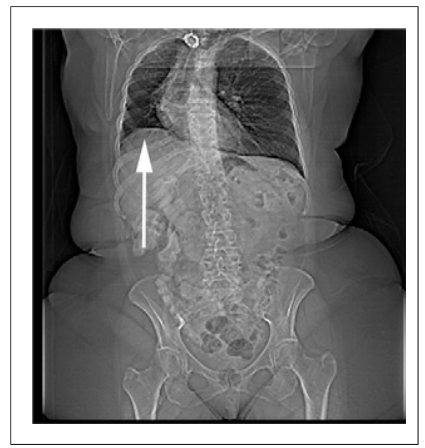

FIGURE 2. Scout diagnostic CT image clearly depicts elevated uptake in right hemidiaphragm (arrow), which is typical for paralysis secondary to phrenic nerve damage.

\section{CONCLUSION}

Unilateral diaphragmatic focal uptake is an uncommon abnormal variant on ${ }^{18} \mathrm{~F}$-FDG PET but one that should be recognized as benign activity. Given that the activity was ipsilateral to the elevated hemidiaphragm in this case, radiation-induced phrenic neuropathy could be suspected with reasonable certainty $(1,2,4)$. Radiation treatments can result in ipsilateral diaphragmatic paresis if the phrenic nerve is included in the treatment field, as often is the case in targeted therapy to the axilla in lymphoma
$(3,5)$. In this patient, the phrenic nerve was likely irradiated because of the tumor's location in the right lung apex, making this a rare instance of radiogenic phrenic neuropathy in a primary lung malignancy. This case also demonstrates the importance of correlation with patient history and other available imaging modalities to characterize focal uptake in unusual locations.

\section{DISCLOSURE}

No potential conflict of interest relevant to this article was reported.

\section{REFERENCES}

1. Cook GJ, Wegner EA, Fogelman I. Pitfalls and artifacts in ${ }^{18}$ FDG PET and PET/ CT oncologic imaging. Semin Nucl Med. 2004;34:122-133.

2. Lin EC, Bhola R. Unilateral diaphragmatic crus uptake on FDG positron emission tomographic imaging. Clin Nucl Med. 2001;26:479.

3. De Vito EL, Quadrelli SA, Montiel GC, Roncoroni AJ. Bilateral diaphragmatic paralysis after mediastinal radiotherapy. Respiration. 1996;63:187-190.

4. Wang Y, Kalra M, Scott J. Compensatory asymmetric hemidiaphragmatic uptake secondary to contralateral hemidiaphragmatic paresis. Clin Nucl Med. 2013; 38:53-55.

5. Avila EK, Goenka A, Fontenla S. Bilateral phrenic nerve dysfunction: a late complication of mantle radiation. J Neurooncol. 2011;103:393-395. 